\title{
Литература
}

1. Зубов А. В., Зубов Н. В., Зубова А.Ф., Мутлу О. В., Стрекопытова М. В. Расчет устойчивости решений дифференичальных уравнений второго порядка с приложсеними. СПб.: СПбГУ, 1999.184 с.

2. Зубов Н. В., Зубова А.Ф. Автоматизаиия проектирования устойчивости и надежности колебательных систем. СПб.: АООТ «Мобильность-плюс», 2010. 355 с.

\section{ПАРАМЕТРИЧЕСКИЕ СВОЙСТВА МНОЖЕСТВ НЕПРИВОДИМОСТИ ЛИНЕЙНЫХ ДИФФЕРЕНЦИАЛЬНЫХ СИСТЕМ}

\author{
Н.А. Изобов ${ }^{1}$, С.А. Мазаник ${ }^{2}$ \\ ${ }^{1}$ Институт математики НАН Беларуси, Сурганова 11, 220072 Минск, Беларусь \\ izobov@im.bas-net.by \\ 2 Белгосуниверситет, факультет прикладной математики и информатики \\ Независимости 4, 220050 Минск, Беларусь \\ smazanik@bsu.by
}

Рассматриваем линейные дифференциальные системы

$$
\dot{x}=A(t) x, \quad x \in \mathbb{R}^{n}, \quad\|A(t)\| \leqslant a<+\infty, \quad t \geqslant 0,
$$

с кусочно-непрерывными ограниченными коэффициентами.

В нашей работе [1] введены непустые так называемые множества неприводимости $N_{2}(a, \sigma)$ и $N_{3}(a, \sigma), \sigma \in(0,2 a]$, всех тех систем $\left(1_{A}\right)$ с матрицами коэффициентов $A$, для каждой из которых существует неприводимая к ней система $\left(1_{B}\right)$ с матрицей коэффициентов $B$, удовлетворяющей соответственно либо условию $\|B(t)-A(t)\| \leqslant C_{B} e^{-\sigma t}, t \geqslant 0$, либо более общему условию $\lambda[B-A] \leqslant-\sigma$. Как уже отмечалось в [1], эти множества связаны очевидным включением $N_{2}(a, \sigma) \subset N_{3}(a, \sigma)$ при $\sigma \in(0,2 a]$ и для них же в работе [2] установлено свойство $N_{3}(a, \sigma) \backslash N_{2}(a, \sigma) \neq \varnothing$ при всех $\sigma \in(0,2 a]$. Кроме того, из результатов работы [3] следует, что эти множества являются непустыми при $\sigma \in(0,2 a]$ и пустыми при $\sigma>2 a$.

Для указанных множеств неприводимости как функций параметров $a$ и $\sigma$ имеют место следуюшие утверждения.

Теорема 1. Для любых $a_{0}>0, \sigma \in\left(0,2 a_{0}\right)$ выполнено

$$
\operatorname{Lim}_{a \rightarrow a_{0}-0} N_{i}(a, \sigma) \neq N_{i}\left(a_{0}, \sigma\right), \quad \operatorname{Lim}_{a \rightarrow a_{0}+0} N_{i}(a, \sigma)=N_{i}\left(a_{0}, \sigma\right), \quad i=2,3 .
$$

Теорема 2. Для любых $a>0, \sigma_{0} \in(0,2 a]$ выполнено

$$
\operatorname{Lim}_{\sigma \rightarrow \sigma_{0}-0} N_{2}(a, \sigma) \neq N_{2}\left(a, \sigma_{0}\right), \quad \operatorname{Lim}_{\sigma \rightarrow \sigma_{0}+0} N_{2}(a, \sigma) \neq N_{2}\left(a, \sigma_{0}\right) .
$$

Теорема 3. Для любых $a>0, \sigma_{0} \in(0,2 a]$ выполнено

$$
\operatorname{Lim}_{\sigma \rightarrow \sigma_{0}-0} N_{3}(a, \sigma)=N_{3}\left(a, \sigma_{0}\right), \quad \operatorname{Lim}_{\sigma \rightarrow \sigma_{0}+0} N_{3}(a, \sigma) \neq N_{3}\left(a, \sigma_{0}\right) .
$$

\section{Литература}

1. Изобов Н. А., Мазаник С.А. Обиий признаж приводимости линейных дифференииальных систем и свойства коэффиииентов приводимости // Дифференц. уравнения. 2007. Т. 43, №2. С. 191202.

2. Изобов Н. А., Мазаник С. А. О множсествах линейных дифференииальных систем, к которым неприводимы возмущенные линейные системы // Дифференц. уравнения. 2011. Т. 47, №11. С. 15451550.

3. Изобов Н. А., Мазаник С. А. Об асимптотически эквивалентных системах при экспоненииалъно убывающих возмущениях // Дифференц. уравнения. 2006. Т. 42, №2. С. 168-174. 